\title{
Non-secretory myeloma in young man mimicking the Gorham disease: case report and the literature review
}

\author{
HANENE LASSOUED FERJANI ${ }^{1,3,4}$, MOALLA MARIEM ${ }^{1}$, HASSEN AFFESS $^{1,2,3}$, KAOUTHER $^{2}$ \\ MAATALLAH ${ }^{1,3,4}$, DHIA KAFFEL ${ }^{1,3,4}$, MOURAD JENZRI ${ }^{1,2,3}$, WAFA HAMDI ${ }^{1,3,4}$
}

\author{
${ }^{1}$ Rheumatology department, Kassab orthopedics institute, Ksar Saïd, Tunisia \\ ${ }^{2}$ Pediatric orthopedics department, Kassab orthopedics institute, Ksar Saïd, Tunisia \\ ${ }^{3}$ Faculty of medicine of Tunis, University Tunis el Manar, Tunisia \\ ${ }^{4}$ Research unit UR17SP04, 2010, Ksar Said 2010, Tunis, Tunisia
}

\begin{abstract}
Multiple myeloma is a neoplasm of plasma cells affecting mostly the elderly with incidence peaks between 60 and 70 years. This disease is exceedingly rare in younger people, especially in adults under 30-year-old. Non-secretory multiple myeloma accounts for $1-5 \%$ of all cases of multiple myeloma. It is also a rare condition in young adult patients, and only six cases have been reported [1]. We herein describe a rare case of non-secretory myeloma in a 22-year-old male, explaining from chest wall pain, without general manifestation. Plain radiography and CT scans revealed diffuse osteolytic lesion mimicking the Gorham disease. A bone marrow biopsy was conducted, revealing the diagnosis of myeloma.
\end{abstract}

Key words: multiple myeloma, Gorham disease, young people, non-secretory.

\section{INTRODUCTION}

Multiple myeloma (MM) is an age-related blood cell malignancy affecting the elderly with a median age at diagnosis of approximately 70 years. Forty percent of patients are older than 75 years [2]. Less than $2 \%$ of $\mathrm{MM}$ are under 40 years. This prevalence reached $0.3 \%$ in individuals younger than 30 years [3]. Clinical course, biological and radiological findings in younger and elderly MM patients were almost similar. However, survival duration and prognosis were more clearly better for younger subjects.

Non-secretory multiple myeloma (NSMM) is an exceedingly rare subset of MM. It represents only $1-5 \%$ of all myeloma and is more exceptional in young adults. Only 6 cases were reported in the literature. Their diagnosis requires bone marrow plasmacytosis detection, in the absence of monoclonal gammopathy in the serum or urine [4].

With this case report, we aim to raise awareness among clinicians about the possibility of MM in young patients.

The exceptional occurrence of NSMM in young adults makes this case worthy of illustration. Prompt disease detection will guarantee earlier therapeutic care and improves the disease prognosis that might be rapidly fatal. To our knowledge, this is the first literature review about non-secretory MM in young patients. Herein we described clinical, biological features, and outcomes of MM in young subjects.

\section{Case presentation}

A 22-year-old male with no significant disease history complained of tenderness in the anterior chest wall and mechanical backache, evolving for seven months. Bone pain was initially diurnal and relieved with paracetamol through the first months of symptoms, worsened within the last month, becoming nocturnal and non-responding to opioids. The patient had no weakness nor fatigue. Physical examination revealed tenderness within the thoracic spine. There was not adenomegaly nor hepatosplenomegaly. The neurological examination was unremarkable.

Laboratory tests showed neither anemia nor any other cytopenia. The peripheral blood smear didn't reveal immature cell or rouleaux formation. Routine laboratory analyses were normal. Creatinine, calcium rate, and phosphate were at $10.4 \mathrm{mg} / \mathrm{L}, 98 \mathrm{mg} / \mathrm{L}$, and $40 \mathrm{mg} / \mathrm{l}$; respectively. 
Inflammatory markers were normal with erythrocyte sedimentation rate at $21 \mathrm{~mm}$, and Creactive protein at $3 \mathrm{mg} / \mathrm{l}$.

A serum proteins electrophoresis showed a slightly decreased level of gamma-globulin at 4.9 $\mathrm{g} / \mathrm{l}$. However, immuno-fixation electrophoresis in blood and urine showed no monoclonal band.

A thoracic computed tomography (CT) scan was performed and revealed a diffused lacunar osteolysis of the entire explored skeleton. The diagnosis of Vanishing bone syndrome was suspected (Figure no.1).

A bone marrow CT-guided biopsy of the left iliac wing was finally performed. Histological examination showed an abundant hemorrhagic background with a marked plasmacytosis. The diagnostic of NSMM was concluded. The patient was referred to a hematologist and subsequently started chemotherapy with Bortezomib-dexamethasone before auto stem cell transplantation.

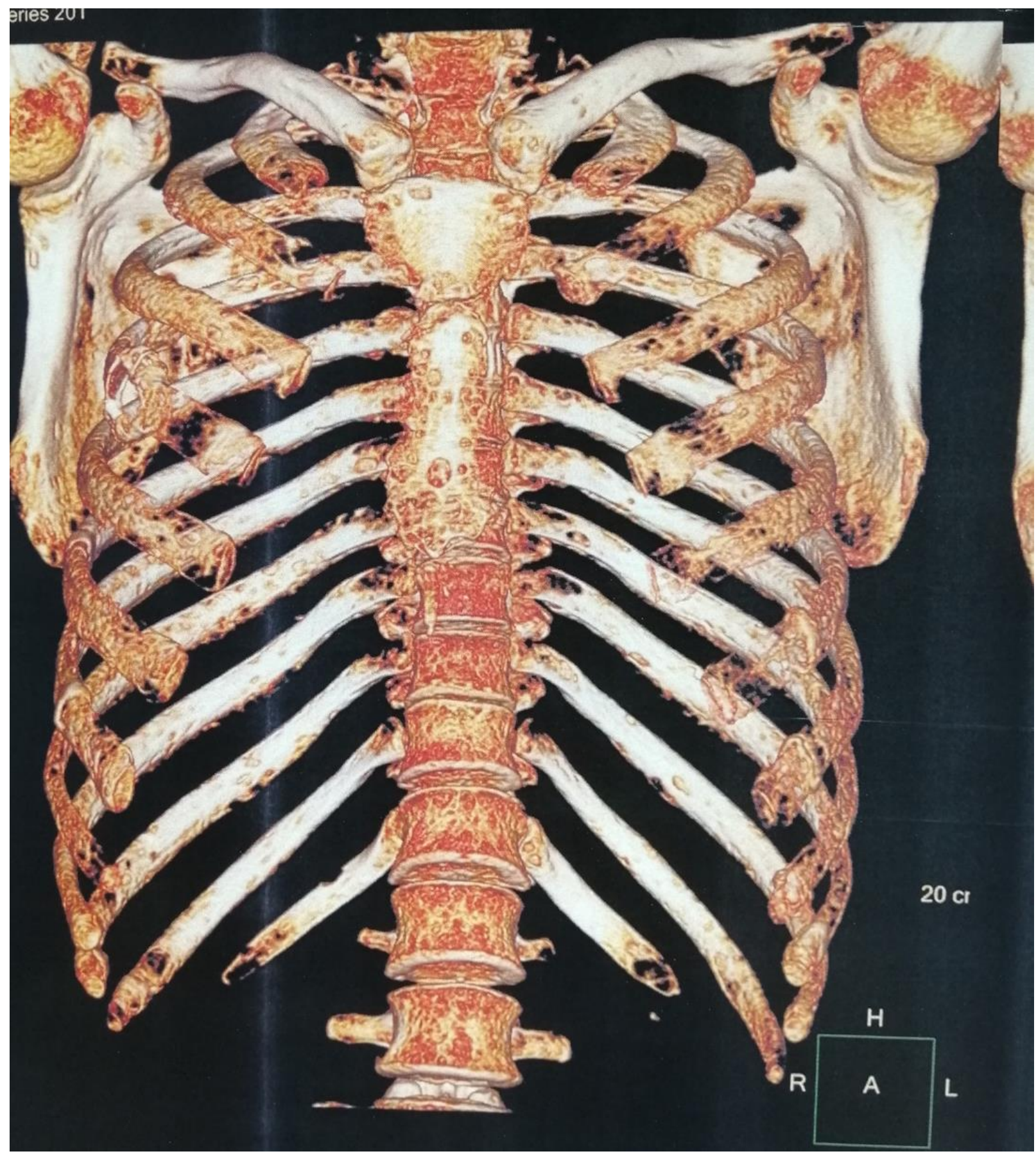

Figure 1. The CT scan showing the multi lacunar osteolytic lesion in sternum, ribs and spine on sagittal (a) and the 3D sequency(b). 


\section{DISCUSSION}

MM is the most common malignant cell disorder in aged patients. It is characterized by the proliferation of neoplastic plasma cells within the bone marrow and the anarchical secretion of monoclonal protein [5].

The frequency of MM in younger adults is exceedingly rare, and $0.3 \%$ of cases occur in persons under 30 years $[6,7]$. While clinical features and outcomes seem similar to the elderly population with MM, there are some differences.

Table 1 summarized cases and series reported in the literature [7-39].

Among 123 patients with MM diagnosed at age under 30, a male predominance was striking, with a male-to-female ratio at $1.27(\mathrm{M}=69)$. This proportion generally decreased with age-onset $[5,7]$. Clinical presentation was commonly silent in younger patients $[6,40]$. On review, the chief presenting complaints in young people were bone pain, as in the current case. However, general symptoms as weakness, weight loss, and fever were less common. Extramedullary involvement may be the first sign of disease and was found in 9 reported cases.

According to the series reported by Bladé and Nakaya, the most common CRAB symptom (hypercalcemia, renal insufficiency, anemia, bone lesion) in young $\mathrm{MM}$ was bone lesions. Its incidence ranges between $69 \%$ and $76 \%$ [7,39]. The contradictory calcemic rate somewhat increased.

Another biologic particularity seen in younger MM in The Jurczyszyn series was the high rate of LDH [34]. However, this finding was not found in our patient.

The $\mathrm{M}$ protein is used as a diagnostic tool for the clone of plasma cell identification, as a tumor marker to follow the course of the MM and response to therapy $[20,41]$. Similar to the elderly, MM the most frequently secreted $M$ protein was $\mathrm{IgG}$, followed by Ig A, and a light chain which was found in $41 \%, 45 \%$, and $21 \%$ of series; respectively [7,39]. Nevertheless, some patients have no detectable immunoglobin within serum or urine. In this subset of MM, the diagnosis was usually challenging, and bone marrow proof of plasma cell proliferation was mandatory. Indeed, NSMM was scarce. Its frequency varies between 1 to $5 \%$ of MM $[4,42]$. In the recent series, patients with NSMM were younger than those with secretory form, have fewer CRAB signs, and had less extensive bone marrow infiltration [2]. Nevertheless, NSMM in under
30 years patients is not recognized. In all reported cases, it was be seen in 6 cases.

The absence of M protein and Bence Jones protein in the present patient delays diagnosis and strengthens the vanishing bone syndrome hypothesis. Interestingly, the imaging features on the chest wall and spine CT scan in our patient revealed diffuse lacunar osteolysis and cortical destruction. These findings mimic a benign osteolytic disease, especially in the absence of $\mathrm{M}$ protein. A similar case was recently reported by Mwania et al., in a 25-year-old female with MM [35]. In this later imaging findings in the appendicular skeleton evoked the vanishing bone disease with the lymphatic vessels' overgrowth [43]. In both of these cases, bone marrow biopsy was the discriminated diagnosis key.

A wide variety of chromosome abnormalities was identified in MM [44]. Any deletion or translocation tends to be specific for early age myeloma. Sagaster et al were compared these chromosomal abnormalities in three groups of MM patients aged <45 years, between 45 and 70 years, and $>70$ years old, and reported that all chromosomal patterns were not significantly different between the three age cohorts [45].

According to the International Staging System (ISS), stages I and II are most frequent at diagnosis time in young MM. However, stage III of the ISS is rare [6,39]. Indeed, $68 \%$ of the patients in the Jurczyszyn cohort were diagnosed in stage I of ISS [34]. Additionally, survival among young MM seems to be longer than older MM, especially in the non-secretory form [42]. This suggestion may be explained by the lack of comorbidities in the younger population and the possibility of stem cell transplantation (SCT) at this age.

Many factors may determine the treatment strategy, especially age, general health, and laboratory results [46]. In the last decade, the new treatment approach has improved outcomes in patients with MM [47]. The chemotherapy pattern used several protocols (table 1).

In the lack of specific protocol in younger myeloma, chemotherapy recommended has often been the same as the adult MM ( $<65$ years). However, young people are eligible for high-dose chemotherapy and autologous SCT due to their age.

SCT was the most used therapeutic strategy in young MM in the Nakaya series [39]. Similar to our case, chemotherapy was initiated waiting for SCT and no accurate statement regarding its prognosis was made. 
Table 1

Literature review: Clinical characteristics and outcomes of young people with plasma cell myeloma

\begin{tabular}{|c|c|c|c|c|c|c|c|c|c|c|c|}
\hline \multirow{2}{*}{$\begin{array}{l}\text { Ref } \\
n^{\circ}\end{array}$} & \multirow{2}{*}{ 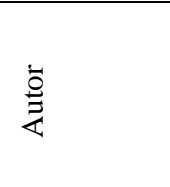 } & \multirow{2}{*}{ 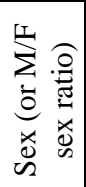 } & \multirow{2}{*}{$\underset{8}{\infty}$} & \multirow{2}{*}{ 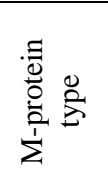 } & \multirow{2}{*}{$\begin{array}{c}\text { Clinical } \\
\text { presentation }\end{array}$} & \multicolumn{4}{|c|}{ CRAB symptoms } & \multirow[t]{2}{*}{ Treatment } & \multirow[t]{2}{*}{ Outcome } \\
\hline & & & & & & $\begin{array}{l}\text { Hyper- } \\
\text { calcemia }\end{array}$ & $\begin{array}{l}\text { Renal } \\
\text { failure }\end{array}$ & Anemia & $\begin{array}{l}\text { Bone } \\
\text { lesion }\end{array}$ & & \\
\hline (8) & $\begin{array}{l}\text { Maeda } \\
\text { and al } \\
1973\end{array}$ & $\mathrm{~F}$ & 13 & IgA & $\begin{array}{l}\text { Breast } \\
\text { masses } \\
\text { with } \\
\text { subcutaneo } \\
\text { us nodules }\end{array}$ & No & $\mathrm{NP}$ & No & No & $\begin{array}{l}\text { Corticosteroids } \\
\text { +gamma- } \\
\text { globulin+ } \\
\text { vincristine }\end{array}$ & Death \\
\hline \multirow[t]{3}{*}{ (9) } & \multirow[t]{3}{*}{$\begin{array}{l}\text { Hewell and } \\
\text { Alexanian } \\
1976\end{array}$} & M & 17 & IgA & $\begin{array}{l}\text { knee pain } \\
\text { and mass in } \\
\text { the } \\
\text { occipital } \\
\text { area }\end{array}$ & NP & NP & No & Yes & $\begin{array}{l}\text { radiotherapy in } \\
\text { bone lesion }\end{array}$ & $\begin{array}{l}\text { Chemothe } \\
\text { rapy not } \\
\text { required }\end{array}$ \\
\hline & & $\mathrm{M}$ & 22 & $\begin{array}{l}\text { IgG- } \\
\text { lambda }\end{array}$ & $\begin{array}{l}\text { lymph } \\
\text { node } \\
\text { enlargemen } \\
\text { t }\end{array}$ & No & No & No & Yes & $\mathrm{MP}+\mathrm{AD}$ & $\begin{array}{l}\text { Indolent } \\
\text { clinical } \\
\text { course }\end{array}$ \\
\hline & & $\mathrm{M}$ & 20 & NS & $\begin{array}{l}\text { Diplopia } \\
\text { and } \\
\text { occipital } \\
\text { mass }\end{array}$ & No & No & No & Yes & CHOP & $\begin{array}{l}\text { Asympto- } \\
\text { matic at } 9 \\
\text { months of } \\
\text { follow up }\end{array}$ \\
\hline (10) & $\begin{array}{l}\text { Clough } \\
\text { et al } \\
1977\end{array}$ & $\mathrm{~F}$ & 24 & IgG-k & $\begin{array}{l}\text { Pallor, } \\
\text { ecchymoses } \\
\text { and renal } \\
\text { failure }\end{array}$ & NP & Yes & Yes & No & $\begin{array}{l}\mathrm{P}+\mathrm{AD}+\mathrm{CA} \\
\text { Then after two } \\
\text { months: } \\
\text { BCNU+MP+C }\end{array}$ & $\begin{array}{l}\text { Death five } \\
\text { months } \\
\text { from } \\
\text { diagnosis }\end{array}$ \\
\hline (11) & $\begin{array}{l}\text { Levin et al } \\
1977\end{array}$ & $\mathrm{~F}$ & 18 & $\mathrm{IgG}$ & $\begin{array}{l}\text { vision } \\
\text { problem } \\
\text { pregnant } \\
\text { woman }\end{array}$ & No & $\mathrm{NP}$ & Yes & Yes & $\begin{array}{l}\text { Local } \\
\text { radiotherapy } \\
\text { MP }\end{array}$ & $\begin{array}{l}\text { Excellent } \\
\text { Response }\end{array}$ \\
\hline \multirow[t]{2}{*}{ (12) } & \multirow[t]{2}{*}{$\begin{array}{l}\text { Lazarus } \\
\text { et al } \\
1980\end{array}$} & $\mathrm{M}$ & 22 & $\mathrm{IgG}$ & Bone pain & $\mathrm{Np}$ & $\mathrm{Np}$ & $\mathrm{Np}$ & Yes & $\begin{array}{l}\text { Cis + BCNU + } \\
\text { CVP + } \\
\text { chlorambucil }\end{array}$ & $\begin{array}{l}\text { No } \\
\text { significant } \\
\text { Improve- } \\
\text { ment }\end{array}$ \\
\hline & & $\mathrm{M}$ & 22 & $\operatorname{IgA}$ & Paraplegia & No & No & No & Yes & $\begin{array}{l}\mathrm{MP}+ \\
\text { radiotherapy }\end{array}$ & Failure \\
\hline (13) & $\begin{array}{l}\text { Bernstein } \\
\text { et al } \\
1985\end{array}$ & $\mathrm{~F}$ & 12 & IgA-k & $\begin{array}{l}\text { nasopharyn } \\
\text { geal mass }\end{array}$ & No & No & No & No & $\begin{array}{l}\mathrm{V}+\mathrm{AD}+\mathrm{P} \text { for } 21 \\
\text { days then } \\
\text { changed to } \mathrm{M}-2 \\
\text { protocole }\end{array}$ & $\begin{array}{l}\text { Stable } \\
\text { IgA level. }\end{array}$ \\
\hline (14) & $\begin{array}{l}\text { Prematillek } \\
\text { et al } \\
1987\end{array}$ & $\mathrm{M}$ & 12 & NP & NP & NP & NP & Yes & Yes & NP & NP \\
\hline (15) & $\begin{array}{l}\text { Harster and } \\
\text { Krause } \\
1987\end{array}$ & $\mathrm{~F}$ & 29 & NS & Post partum & $\mathrm{NP}$ & $\mathrm{NP}$ & Yes & Yes & $\mathrm{NP}$ & $\mathrm{NP}$ \\
\hline (16) & $\begin{array}{l}\text { Rapoport } \\
\text { and Rowe } \\
1990\end{array}$ & M & 15 & IgA-k & $\begin{array}{l}\text { Bone pain } \\
\text { Neurologic } \\
\text { al } \\
\text { symptoms }\end{array}$ & NP & $\mathrm{NP}$ & NP & Yes & $\begin{array}{l}\text { CVP+M } \\
\text { BCNU }\end{array}$ & $\begin{array}{l}\text { Good } \\
\text { response }\end{array}$ \\
\hline (17) & $\begin{array}{l}\text { Hermann } \\
\text { et al } \\
1990\end{array}$ & $\mathrm{~F}$ & 28 & $\operatorname{IgD}$ & $\begin{array}{l}\text { pain in the } \\
\text { anterior wall } \\
\text { of the chest, } \\
\text { thoracic } \\
\text { spine, and } \\
\text { right } \\
\text { shoulder }\end{array}$ & - & - & - & Yes & NP & NP \\
\hline
\end{tabular}




\begin{tabular}{|c|c|c|c|c|c|c|c|c|c|c|c|}
\hline (18) & $\begin{array}{l}\text { Pajor } \text { et al } \\
1991\end{array}$ & $\mathrm{~F}$ & 27 & IgG-1 & $\begin{array}{l}\text { Anemia } \\
\text { Pregnant } \\
\text { woman }\end{array}$ & No & No & Yes & NP & $\begin{array}{l}\text { interferon-a } \\
\mathrm{MP}+\mathrm{V}\end{array}$ & $\begin{array}{l}\text { Partial } \\
\text { remission, } \\
2 \text { years after } \\
\text { treatment }\end{array}$ \\
\hline (19) & $\begin{array}{l}\text { Kaste } \text { et al } \\
1992\end{array}$ & $\mathrm{M}$ & 15 & IgG & bone pain & No & NP & Yes & Yes & NP & NP \\
\hline (20) & $\begin{array}{l}\text { Grunwald } \\
\text { et al } \\
1993\end{array}$ & $\mathrm{M}$ & 25 & IgG-1 & - & - & - & Yes & Yes & NP & NP \\
\hline (21) & $\begin{array}{l}\text { Badwey } \\
\text { et al } \\
1993\end{array}$ & $\mathrm{~F}$ & 25 & IgG-k & $\begin{array}{l}\text { Knee pain } \\
\text { and } \\
\text { pathological } \\
\text { fracture }\end{array}$ & NP & NP & No & Yes & Radiotherapy & $\begin{array}{l}\text { Stable } \\
\text { after } 3 \\
\text { years }\end{array}$ \\
\hline (22) & $\begin{array}{l}\text { Ishida and } \\
\text { Dorfman } \\
\text { 1995 }\end{array}$ & $\mathrm{F}$ & 23 & NS & $\begin{array}{l}\text { lump of the } \\
\text { head }\end{array}$ & $\mathrm{NP}$ & NP & NP & Yes & NP & NP \\
\hline (7) & $\begin{array}{l}\text { Blade et al } \\
1996\end{array}$ & $\begin{array}{l}6 \mathrm{M} \\
4 \mathrm{~F}\end{array}$ & in & $\begin{array}{l}\text { light } \\
\text { chain } \\
(5) \\
\operatorname{IgD}(2) \\
\operatorname{IgG}(2)\end{array}$ & $\begin{array}{l}\text { Extra- } \\
\text { medullary } \\
\text { involvement } \\
\text { (4) }\end{array}$ & $\begin{array}{l}\text { Yes } \\
\text { (2) }\end{array}$ & $\begin{array}{l}\text { Yes } \\
\text { (3) }\end{array}$ & Yes (2) & $\begin{array}{l}\text { Yes } \\
(6)\end{array}$ & $\begin{array}{l}\text { radiation } \\
\text { therapy (4) } \\
\text { MP (6) } \\
\text { Second line } \\
\text { chemiotherapy } \\
\text { (6) }\end{array}$ & Death (7) \\
\hline (23) & $\begin{array}{l}\text { Dubrey } \\
\text { et al } \\
2008\end{array}$ & $\mathrm{~F}$ & 29 & IgG-K & $\begin{array}{l}\text { vomiting } \\
\text { and bone } \\
\text { pain }\end{array}$ & Yes & Yes & Yes & Yes & $\begin{array}{l}\text { CTD+dexa } \\
\text { Auto-SCT }\end{array}$ & NP \\
\hline (24) & $\begin{array}{l}\text { Okoli } \\
2009\end{array}$ & $\mathrm{~F}$ & 28 & $\operatorname{IgA}$ & $\begin{array}{l}\text { Abdominal } \\
\text { pain and } \\
\text { vomiting } \\
\text { weight loss }\end{array}$ & NP & $\mathrm{Np}$ & Yes & No & $\mathrm{NP}$ & NP \\
\hline (25) & $\begin{array}{l}\text { Crusoe } \\
\text { et al } \\
2015\end{array}$ & $\mathrm{M}$ & 8 & IgG-k & back pain & No & No & Yes & NP & $\begin{array}{l}3 \text { cycles of } \\
\text { VCD } \\
3 \text { cycles } \\
\text { DTPACE } \\
\text { high-dose M } \\
\text { auto SCT } \\
\end{array}$ & $\begin{array}{l}\text { Failure } \\
\text { Failure } \\
\text { Partial } \\
\text { response } \\
\text { Partial } \\
\text { response }\end{array}$ \\
\hline (26) & $\begin{array}{l}\text { Akhtar } \\
\text { and al } \\
2005\end{array}$ & $\mathrm{~F}$ & 30 & & $\begin{array}{l}\text { weakness } \\
\text { and pain }\end{array}$ & No & No & No & Yes & MP & $\begin{array}{l}\text { Improve- } \\
\text { ment after } \\
6 \text { cycles of } \\
\text { chemo- } \\
\text { therapy }\end{array}$ \\
\hline (27) & $\begin{array}{l}\text { El Mangad } \\
\text { and al } \\
2014\end{array}$ & M & 27 & $\begin{array}{l}\text { BJP } \\
\text { lambd } \\
\text { a light } \\
\text { chain } \\
\end{array}$ & bone pain & Yes & yes & Yes & Yes & $\begin{array}{l}\text { CTD+dexa } \\
\text { SCT }\end{array}$ & \\
\hline (28) & $\begin{array}{l}\text { Pilbeam } \\
\text { and al } \\
2017 \\
\end{array}$ & $\mathrm{M}$ & 14 & IgA-k & $\begin{array}{l}\text { low back } \\
\text { pain }\end{array}$ & No & No & No & Yes & $\begin{array}{l}\text { Radiotherapy } \\
\text { systemic } \\
\text { therapy (NP) }\end{array}$ & $\begin{array}{l}\text { Good } \\
\text { response }\end{array}$ \\
\hline (29) & $\begin{array}{l}\text { Radhakrish } \\
\text { nan and al } \\
2017\end{array}$ & $\mathrm{M}$ & 8 & IgM & $\begin{array}{l}\text { diffuse } \\
\text { body pain, } \\
\text { vomiting, } \\
\text { and fever }\end{array}$ & Yes & No & Yes & Yes & $\mathrm{P}$ & Death \\
\hline (30) & $\begin{array}{l}\text { Kumar } \\
\text { et al } \\
2017 \\
\end{array}$ & $\mathrm{~F}$ & 28 & IgG & $\begin{array}{l}\text { Extramedul } \\
\text { lary mass }\end{array}$ & NP & NP & Yes & Yes & $\begin{array}{l}\text { surgery } \\
\text { followed by } \\
\text { chemo-radiation }\end{array}$ & $\begin{array}{l}\text { Death } \\
\text { after } \\
6 \text { months } \\
\end{array}$ \\
\hline (31) & $\begin{array}{l}\text { Webber } \\
\text { et al } 2017\end{array}$ & $\mathrm{M}$ & 25 & $\begin{array}{l}\text { BJP } \\
\text { kappa } \\
\text { free } \\
\text { light } \\
\text { chains }\end{array}$ & $\begin{array}{l}\text { infectious } \\
\text { complicatio } \\
n\end{array}$ & Yes & No & Yes & Yes & $\begin{array}{l}\text { VRd therapy } \\
\text { Auto SCT }\end{array}$ & $\begin{array}{l}\text { Remission } \\
\text { after } \\
1 \text { year }\end{array}$ \\
\hline (32) & $\begin{array}{l}\text { Bellahammou } \\
\text { et al } 2018\end{array}$ & $F$ & 25 & $\begin{array}{l}\text { IgG-k } \\
\text { and } \\
\text { BJP } \\
\end{array}$ & $\begin{array}{l}\text { Bone pain } \\
\text { Asthenia }\end{array}$ & No & No & Yes & Yes & VAD & NP \\
\hline
\end{tabular}




\begin{tabular}{|c|c|c|c|c|c|c|c|c|c|c|c|}
\hline (33) & $\begin{array}{l}\text { Mufti and } \\
\text { Marathe } \\
2018\end{array}$ & $\mathrm{~F}$ & 20 & $\operatorname{Ig} \mathrm{A}$ & $\begin{array}{l}\text { nausea, } \\
\text { vomiting, } \\
\text { fatigue, } \\
\text { hemorrhages }\end{array}$ & NP & Yes & Yes & No & CyBorD & $\begin{array}{l}\text { Improve- } \\
\text { ment } \\
\text { of renal } \\
\text { function }\end{array}$ \\
\hline (34) & $\begin{array}{l}\text { Artur } \\
\text { and al } \\
2018\end{array}$ & $\begin{array}{l}35 \mathrm{M} \\
17 \mathrm{~F}\end{array}$ & $\begin{array}{l}\text { के } \\
\dot{c} \\
\infty \\
\infty \\
\text { d }\end{array}$ & & & 6 & 4 & 13 & 36 & $\begin{array}{l}\text { No therapy } 11 \\
\text { Conventional } \\
\text { chemotherapy (4) } \\
\text { Chemotherapy+/- } \\
\text { immunomodulat } \\
\text { or +/- } \\
\text { proteasome } \\
\text { inhibitor (29) }\end{array}$ & $\begin{array}{l}5 \text {-year OS } \\
\text { rate of } \\
77 \%\end{array}$ \\
\hline (35) & $\begin{array}{l}\text { Mwania } \\
\text { et al } \\
2019 \\
\end{array}$ & $\mathrm{~F}$ & 25 & IgG-k & $\begin{array}{l}\text { Neurologic } \\
\text { al } \\
\text { symptoms }\end{array}$ & No & No & Yes & Yes & $\begin{array}{l}\text { CyBorD } \\
\text { radiotherapy }\end{array}$ & $\begin{array}{l}\text { Good } \\
\text { response }\end{array}$ \\
\hline (36) & $\begin{array}{l}\text { Van Man } \\
\text { H } 2019\end{array}$ & $\mathrm{~F}$ & 17 & $\begin{array}{l}\mathrm{IgG} \\
\text { lambda }\end{array}$ & Bone pain & NP & $\mathrm{NP}$ & $\mathrm{NP}$ & Yes & $\begin{array}{l}\text { B and dexa } \\
\text { ASCT }\end{array}$ & $\begin{array}{l}\text { Good } \\
\text { response }\end{array}$ \\
\hline (37) & $\begin{array}{l}\text { Khan } \\
\text { and al } \\
2020 \\
\end{array}$ & $\mathrm{~F}$ & 26 & NP & $\begin{array}{l}\text { Fever } \\
\text { Bone pain }\end{array}$ & Yes & Yes & Yes & Yes & CyBorD & $\begin{array}{l}\text { Good } \\
\text { response }\end{array}$ \\
\hline (38) & $\begin{array}{l}\text { Lee } \text { et al } \\
2020\end{array}$ & $\mathrm{~F}$ & 24 & IgG-1 & $\begin{array}{l}\text { Neurologic } \\
\text { al symptom }\end{array}$ & $\mathrm{NP}$ & $\mathrm{NP}$ & $\mathrm{NP}$ & Yes & $\begin{array}{l}\text { CyBorD } \\
\text { C+P+E+Cis+DA }\end{array}$ & $\begin{array}{l}\text { Failure } \\
\text { Good } \\
\text { response }\end{array}$ \\
\hline (39) & $\begin{array}{l}\text { Nakaya } \\
\text { et al } \\
2020\end{array}$ & $2 / 1$ & 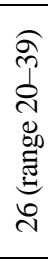 & $\begin{array}{l}\operatorname{IgG} \\
(11) \\
\mathrm{BJP}(9) \\
\operatorname{IgA}(3) \\
\mathrm{NS}(2) \\
\operatorname{IgD} \\
(1)\end{array}$ & Bone pain & Yes (1) & $\begin{array}{l}\text { Yes } \\
\text { (11) }\end{array}$ & Yes (6) & $\begin{array}{l}\text { Yes } \\
\text { (18) }\end{array}$ & $\begin{array}{l}\text { auto-SCT } 10 \\
\text { auto-SCT + } \\
\text { allo-SCT(6) } \\
\text { chemotherapy } \\
\text { without } \\
\text { SCT (5) } \\
\text { allo-SCT (5) }\end{array}$ & Death (12) \\
\hline
\end{tabular}

MP: melphalan and prednisone, CHOP: vincristine, adriamycin, cyclophosphamide, and prednisone; BCNU: carmustine; CVP: cyclophosphamide, vincristine and prednisone, CTD: cyclophosphamide and thalidomide, VCD: cyclophosphamide, bortezomib, and dexamethasone; M2 protocole: vincristine $+B C N U$ +cyclophosphamide+melphalan +prednisolone; DTPACE: dexamethasone, thalidomide, cisplatin, doxorubicin, cyclophosphamide, and etoposide), VRd therapy: bortezomib-lenalidomide-dexamethasone ; VAD: vincristine, doxorubicin, and dexamethasone; CyBorD: Cyclophosphamide,bortezomib and dexamethasone

AD: adriamycine (doxorubicin); CA: cytosine arabinoside (Cytarabine); B: Bortezomib; C: cyclophosphamide; Cis: cisplatin; D: doxorubicin; DA:daratumumab; E:etoposide; M: melphalan; P: prednisolone; MP: melphalan and prednisolone; BJP bence jones protein, NP : not precised; NS: non secretory; SCT: stem cell transplantation; T: thalidomide ; V: vincristine

\section{CONCLUSION}

NSMM is a rare entity in young patients ( $<30$ years). A sternal puncture or a bone marrow biopsy is mandatory when any biological or radiographic signs evoke a $\mathrm{MM}$. In front of alarming symptoms such as osteolytic lesions or hypogammaglobulinemia, the clinician must strive to depict marrow bone infiltration with malignant cells, even in the absence of blood and urinary secretion.

Mielomul multiplu este o neoplazie a plasmocitelor ce afectează populația vârstnică, având un vârf de incidență între 60 și 70 de ani. Este foarte rară la pacienții tineri, mai ales la cei sub 30 de ani. Mielomul multiplu non-secretor reprezintă 1-5\% din totalul mieloamelor și au fost raportate numai 6 cazuri în literatură. Prezentăm cazul unui pacient de 22 de ani, cu durere toracică și fără manifestări generale. CT-ul vizualizează leziuni difuze osteolitice ce mimează boala Gorham. Puncția biopsie medulară a tranșat diagnosticul de mielom.

Correspondence to: Lassoued Ferjani Hanène, M.D, Rheumatology department, Kassab orthopedics institute, Ksar Saïd 2010 , Tunisia

E-mail: lassouedferjanihanene@gmail.com, Phone: 0021697950842

Conflict of interest disclosure: The authors dclare no conflict of interest. 


\section{REFERENCES}

1. USHA N., AGARWAL N., KUMAR P., RAI M., SINGH RG., SETH M., et al. Myeloma in young age. Indian J Pathol Microbiol. 2005;48(3):314-7.

2. MIGKOU M., AVIVI I., GAVRIATOPOULOU M., COHEN YC., FOTIOU D., KANELLIAS N., et al. Clinical characteristics and outcomes of oligosecretory and non-secretory multiple myeloma. Ann Hematol. 2020;99(6):1251-5.

3. BLADÉ J., KYLE RA., GREIPP PR. Presenting features and prognosis in 72 patients with multiple myeloma who were younger than 40 years. Br J Haematol.1996;93(2):345-51.

4. CORSO A., MANGIACAVALLI S. Non-Secretory Myeloma: Ready for a new Definition? Mediterr J Hematol Infect Dis [Internet]. 2017;9(1).

5. KEHRER M., KOOB S., STRAUSS A., WIRTZ DC., SCHMOLDERS J. [Multiple Myeloma - Current Status in Diagnostic Testing and Therapy]. Z Orthop Unfall. 2017;155(5):575-86.

6. CHEEMA PK., ZADEH S., KUKRETI V., REECE D., CHEN C., TRUDEL S., et al. Age 40 years and under does not confer superior prognosis in patients with multiple myeloma undergoing upfront autologous stem cell transmplant. Biol Blood Marrow Transplant. 2009;15(6):686-93.

7. BLADE J., KYLE RA., GREIPP PR. Multiple myeloma in patients younger than 30 years. Report of 10 cases and review of the literature. Arch Intern Med. 1996;156(13):1463-8.

8. MAEDA K., ABESAMIS CM., KUHN LM., HYUN BH. Multiple myeloma in childhood: report of a case with breast tumors as a presenting manifestation. Am J Clin Pathol. 1973;60(4):552-8.

9. HEWELL GM., ALEXANIAN R. Multiple Myeloma in Young Persons. Ann Intern Med. 1976;84(4):441-3.

10. CLOUGH V., DELAMORE IW., WHITTAKER JA. Multiple Myeloma in a Young Woman. Ann Intern Med. 1977;86(1):117-8.

11. LEVIN SR., SPAULDING AG., WIRMAN JA. Multiple Myeloma: Orbital Involvement in a Youth. Arch Ophthalmol. 1977;95(4):642-644.

12. LAZARUS HM., KELLERMEYER RW., AIKAWA M., HERZIG RH. Multiple myeloma in young men clinical course and electron microscopic studies of bone marrow plasma cells. Cancer. 1980;46(6):1397-400.

13. BERNSTEIN SC., PEREZ-ATAYDE AR., WEINSTEIN HJ. Multiple myeloma in a child. Cancer. 1985;56(8):2143-7.

14. PREMATILLEKE MN. Multiple myeloma in a child. Ceylon Med J. 1987;32(3):143-5.

15. HARSTER GA., KRAUSE JR. Multiple myeloma in two young postpartum women. Arch Pathol Lab Med. 1987;111(1):38-42.

16. RAPOPORT AP., ROWE JM. Plasma cell dyscrasia in a 15-year-old boy: case report and review of the literature. Am J Med. 1990;89(6):816-8.

17. HERMANN G., ABDELWAHAB IF., BERSON BD., GREENBERG ML., PALESTRO CJ. Multiple myeloma (IgD) in a 28-year-old woman. Skeletal Radiol. 1990;19(5):379-81.

18. PAJOR A., KELEMEN E., MOHOS Z., HAMBACH J., VÁRADI G. Multiple myeloma in pregnancy. Int. J. Gynecol. Obstet. 1991;35(4):341-2.

19. KASTE SC., RIPPS LG., PEDROSA F., RIBEIRO RC. Multiple myeloma in an adolescent. Pediatr Radiol. 1992;22(4):293-5.

20. GRUNWALD HW., ROSNER F., KALMAN AC. Immunoglobulin-A plasma cell dyscrasia in a young Hispanic man. N Y State J Med. 1993;93(2):146.

21. BADWEY TM., MURPHY DA., EYSTER RL., Cannon MW. Multiple myeloma in a 25-year-old woman. Clin Orthop Relat Res. 1993;(294):290-3.

22. ISHIDA T., DORFMAN HD. Plasma cell myeloma in unusually young patients: a report of two cases and review of the literature. Skeletal Radiol. 1995;24(1):47-51.

23. DUBREY SW., PATEL K. Myeloma in a very young woman presenting with bone pain. Br J Hosp Med (Lond). 2008;69(1):48-9.

24. OKOLI K., IRANI F., HORVATH W. Multiple myeloma and systemic lupus erythematosus in a young woman. J Clin Rheumatol. 2009;15(6):292-4.

25. CRUSOE EDE Q., DA SILVA AM., AGARENO J., CHAUFFAILlE MDE L., BONFIM C., MORAES HUNGRIA VT. Multiple myeloma: a rare case in an 8-year-old child. Clin Lymphoma Myeloma Leuk. 2015;15(1):e31-e33.

26. AKHTAR K., LAGHARI NA., HAQ AU., ANEES M., REHMAN SU., ALAM MI. Multiple myeloma in younger age. J Coll Physicians Surg Pak. 2009;19(1):62-63.

27. MANGAD F.,BOUCHTI I. Multiple Myeloma in Unusually Young Patient: A Case Report. Int J Clin Med, 5, 890-893.

28. PILBEAM KL., LUND TC. Pediatric multiple myeloma. Blood. 2017;129(3):395.

29. RADHAKRISHNAN V., REDDY P., TOTADRI S., SUNDERSINGH S., SAGAR TG. Multiple Myeloma in an 8-Year-Old Child With HIV Infection. Pediatr. Hematol. Oncol. J. 2017;39(1):77

30. KUMAR S., BHUTANI N., BHARGAWA S., KATARIA SP., SEN R. Extra-skeletal plasmablastic myeloma presenting as palatal growth - An unusual entity. Int J Surg Case Rep. 2017;41:423-426.

31. WEBBER T., LAWLOR M., BALACH T. Streptococcus pneumoniae Osteomyelitis in a 25-Year-Old Man as the Initial Presentation of Multiple Myeloma: A Case Report. JBJS Case Connector. 2017;7(3):e72-e72.

32. BELLAHAMMOU K., LAKHDISSI A., AKKAR O., SALMI N., ZAKKOURI F., DAHRAOUI S., et al. Aggressive multiple myeloma in a young adult: a case report. Int. J. Surg. Med. 2017;3(2):1

33. MUFTI M., MARATHE O. A Rare Case of Early Onset Multiple Myeloma in a 20-Year-Old Female With Factor X Inhibitor. J Hematol. 2018;7(2):69-71.

34. JURCZYSZYN A., DAVILA J., KORTÜM KM., JAYABALAN DS., VIJ R., FIALA M., Et al. Multiple myeloma in patients up to 30 years of age: a multicenter retrospective study of 52 cases. Leuk Lymphoma. 2019;60(2):471-6.

35. MWANIA M., KARIM N., WAMBUI S., MOHAMMEDALI S., NJAU A. Plasma cell myeloma lytic lesions mimicking vanishing bone syndrome in a young patient. BJR Case Rep. 2019;5(4):20190025. 
36. VAN MAN H., DUNG PC. A Case of Multiple Myeloma in a 17-Year-Old Girl Treated with Autologous Hematopoietic Stem Cell Transplantation (ASCT). Am J Case Rep. 2019;20:1623-9.

37. KHAN AW., KHAN AA., ALMAS T., ISHAQ M., ULLAH I. Far and Few Between: Early Onset Multiple Myeloma in a 26-Year-Old Female. Cureus. 2020;12(8):e9588.

38. LEE M., ZHANG X., NGUYEN V., Hartley-Brown M. Multiple Myeloma in a Young Female Presenting with Neurological Symptoms. Case Rep Hematol. 2020;2020:1375174.

39. NAKAYA A., KOHARA T., SHIBAYAMA H., ONDA Y., KANDA J., KANEKO H., et al. Retrospective multi-center study of Adolescent and Young Adult (AYA) Multiple Myeloma in Kansai Myeloma Forum registry. Int J Hematol. 2020;112(4):435-8.

40. BLADÉ J., KYLE RA. Multiple Myeloma in Young Patients: Clinical Presentation and Treatment Approach. Leuk Lymphoma. 1998;30(5-6):493-501.

41. WILLRICH MAV., KATZMANN JA. Laboratory testing requirements for diagnosis and follow-up of multiple myeloma and related plasma cell dyscrasias. Clin Chem Lab Med. 2016;54(6):907-19.

42. CHAWLA SS., KUMAR SK., DISPENZIERI A., GREENBERG AJ., LARSON DR., KYLE RA., LACY MQ., GERTZ MA., RAJKUMAR SV. Clinical course and prognosis of non-secretory multiple myeloma. Eur J Haematol. 2015;95(1):57-64.

43. RICCI KW., HAMMILL AM., MOBBERLEY-SCHUMAN P., NELSON SC., BLATT J., BENDER JLG., et al. Efficacy of systemic sirolimus in the treatment of generalized lymphatic anomaly and Gorham-Stout disease. Pediatr Blood Cancer. 2019;66(5):e27614.

44. FONSECA R., BLOOD E., RUE M., HARRINGTON D., OKEN MM., KYLE RA., Et al. Clinical and biologic implications of recurrent genomic aberrations in myeloma. Blood. 2003;101(11):4569-75.

45. SAGASTER V., KAUFMANN H., ODELGA V., ACKERMANN J., GISSLINGER H., RABITSCH W., et al. Chromosomal abnormalities of young multiple myeloma patients $(<45 \mathrm{yr}$ ) are not different from those of other age groups and are independent of stage according to the International Staging System. Eur J Haematol. 2007;78(3):227-34.

46. PALUMBO A., BRINGHEN S., LUDWIG H., DIMOPOULOS MA., BLADE J., MATEOS MV., et al. Personalized therapy in multiple myeloma according to patient age and vulnerability: a report of the European Myeloma Network (EMN). Blood. 2011;118(17):4519-29.

47. ESLICK R., TALAULIKAR D. Multiple myeloma: from diagnosis to treatment. Aust Fam Physician. 2013;42(10):684-8.

Received $11^{\text {th }}$ May 2021 